\title{
MAN.I I - Prospection studies: a new methodology for strengthening research projects conducted by graduate students.
}

\author{
Juliana Santos-Gandelman ${ }^{1 \star}$; Fabricia Pires Pimenta ${ }^{1}$. \\ 1Fiocruz/CTDS.
}

Introduction: Prospecting studies provide an overview of patent documents and scientific literature of a particular technology, being relevant in policy discussions, research strategy planning or technology transfer. The Escritório de Inovação - EI (Innovation Office), of the Centro de Desenvolvimento Tecnológico em Saúde - CDTS, proposed to carry out prospecting studies to strengthen internal research projects conducted mainly by graduate students.

Objective: The present study aimed to provide a methodology to perform an analysis of the scientific and patent literature for postgraduation research projects. The main objective was to give information about the state-of-the-art of the technology, providing inputs for correct decision making, taking into account technological development and innovation.

Methodology: The project "Functional characterization of a panel of tumor-specific aptamers for triple negative breast cancer" served as a pilot. Patent document searches were carried out in September 2018 using the database Orbit Intelligence. We searched for documents containing the words "aptamer* and breast cancer" in their title, abstract, or claims and classified as A61 (human needs - medical or veterinary science; hygiene) by IPC. Regarding the scientific literature, three different databases were used: Web of Science, Scopus and Pubmed with the same key words for patent search. Parts of the study were carried out jointly with the student.

Results: The search resulted in 653 patent families and 753 different scientific papers. Since the first patent filing in 1995, there has been a notable increase in the number of patent deposits involving aptamer technology and its application to breast cancer, with a peak in 2015 . Through the analysis of the countries where assignees sought protection for their inventions, it is possible to infer that the markets of greatest interest in this technology are USA, Europe and China. The majority of the patent documents analyzed is related to the use of aptamers for therapy against breast cancer. Among the analyzed scientific articles, an expansion of the studies involving this technological area was also observed, with most of the research centers located in the USA and China. Through self-correlation analysis, it was observed some networks of interaction between the affiliations of the first authors of the studies analyzed. In relation to Brazil, attention is drawn to the position of the University of the State of Rio de Janeiro as a bridge between national and international institutions.

Conclusion: The prospective analysis can contrisute to many scientific and economical aspects in the research and technological development of aptamers and their use for breast cancer. The designed methodology generates studies suitable to support graduate projects, avoiding waste or replication of scientific research, mainly at public institutions, and can promote the literacy of postgraduation students.

Keywords: technological information; graduate courses; prospective studies 